\title{
Vaginal cuff length during radical hysterectomy is a prognostic factor for stage IB-IIA cervical cancer: a retrospective study
}

This article was published in the following Dove Press journal: Cancer Management and Research

$\mathrm{Na} Z u o,{ }^{1}, *$ Hongzhen $\mathrm{Hu},{ }^{1,2, *}$ Niresh Thapa, ${ }^{1,3}$ Zhen Li, Daqiong Jiang,' Xiangyu Meng, ${ }^{4}$ Jing Yang, ' Xiaoxing Chen, ${ }^{5}$ Hongbing Cai'

'Department of Gynecological Oncology, Zhongnan Hospital of Wuhan University, Hubei Cancer Clinical Study Center, Hubei Key Laboratory of Tumor Biological Behaviors, Wuhan 43007I, Hubei, People's Republic of China; ${ }^{2}$ Department of Obstetrics and Gynecology, Shenzhen Futian Maternity and Child Care Hospital, Shenzhen 518030, Guangdong, People's Republic of China; ${ }^{3}$ Karnali Academy of Health Sciences, Jumla, Nepal; ${ }^{4}$ Center for Evidence-Based and Translational Medicine, Zhongnan Hospital of Wuhan University, Department of Evidence-Based Medicine and Clinical Epidemiology, Second Clinical College of Wuhan University, Wuhan 43007I, Hubei, People's Republic of China; ${ }^{5}$ Department of Gastroenterology, Zhongnan Hospital of Wuhan University, Hubei Cancer Clinical Study Center, Hubei Key Laboratory of Intestinal and Colorectal Diseases, Wuhan 43007I, Hubei, People's Republic of China

*These authors contributed equally to this work

Correspondence: Hongbing Cai Department of Gynecological Oncology, Zhongnan Hospital of Wuhan University, No 169 Donghu Road, Wuchang District, Wuhan 43007I, Hubei, People's Republic of China

Tel +86 I3397।6 8990

Email chb2105@163.com
Purpose: The aim of this study was to investigate the impact of vaginal cuff length (VCL) resected during radical hysterectomy $(\mathrm{RH})$ on the long-term survival outcomes of patients with cervical cancer (International Federation of Gynecology and Obstetrics [FIGO] stage IB-IIA) and to explore whether VCL is a prognostic factor for cervical cancer.

Methods: A total of 435 eligible patients with stage IB and IIA cervical cancer who underwent RH in Zhongnan Hospital of Wuhan University (Wuhan, People's Republic of China) from January 2007 to February 2017 were retrospectively analyzed. These patients were divided into two groups (VCL $\leq 2.0 \mathrm{~cm}$ and $>2.0 \mathrm{~cm}$ ) for further analysis according to the VCL during RH. The Kaplan-Meier method was adopted to calculate the survival rates. Univariate and multivariate Cox regression models were used to analyze independent prognosis factors for survival.

Results: Of the 435 identified patients, 196 had VCL $\leq 2.0 \mathrm{~cm}$ and 239 had VCL $>2.0 \mathrm{~cm}$ after RH. The 5-year disease-free survival (DFS) for the group who had VCL $\leq 2.0 \mathrm{~cm}$ vs the group who had VCL $>2.0 \mathrm{~cm}$ was $68.1 \%$ vs $87.5 \%$ ( $P<0.001)$. Correspondingly, the overall survival (OS) for the two groups was $71.4 \%$ vs $89.2 \%(P<0.001)$. More interestingly, the VCL was significantly associated with the 5-year local recurrence rate, but not associated with the distant metastasis rate. In addition to the VCL, FIGO stage and lymph node involvement were also identified as significant prognostic factors for cervical cancer.

Conclusion: Resection of VCL $>2.0 \mathrm{~cm}$ in RH has a more favorable long-term outcome than VCL $\leq 2.0 \mathrm{~cm}$ among patients with cervical cancer (FIGO stage IB-IIA); shorter VCL resection was significantly associated with local recurrence, DFS, and OS; thus, it can be considered as a prognostic factor for cervical cancer.

Keywords: cervical cancer, vaginal cuff length, lymph node involvement, prognosis, local recurrence, distant metastasis

\section{Introduction}

Cervical cancer is the fourth most frequent malignancy and one of the leading causes of cancer-related death in women, with 527,600 new cases and 265,700 deaths annually worldwide. ${ }^{1,2}$ In People's Republic of China alone, there are around 19\% $(98,900$ cases) new cases and 12\% (30,500 cases) deaths of the total world's cervical cancer burden annually. ${ }^{3}$ According to the FIGO staging system, about $40 \%$ of patients with cervical cancer are diagnosed at early disease stage (stage IA-IIA). ${ }^{4,5}$ Currently, surgical treatment by RH with bilateral pelvic lymph node dissection is an effective treatment modality for these patients. ${ }^{6}$

Since the first publication of a large series of surgeries for cervical cancer by Wertheim in $1912,{ }^{7}$ and subsequently by Okabayashi and Meigs, many radical 
procedures with different degrees of radicality have been described; thereafter, significant progress has been made in the surgical management of cervical cancer. ${ }^{7,8}$ The salient part of $\mathrm{RH}$ is the removal of the lateral parametrium and the upper part of the vagina.

According to Piver et al classification published in 1974, the extent of vaginal resection during RH was recommended to be three-fourths of the vagina; ${ }^{9}$ however, there was lack of good supporting evidence for this recommendation. ${ }^{10}$ Based on a lateral extension of resection, Querleu and Morrow described four types (Type A to Type D) of RH in $2008,{ }^{8}$ and updated the Querleu-Morrow classification in 2017; the authors also suggested that removal of approximately $10 \mathrm{~mm}$ of the tumor-free vaginal cuff is sufficient. ${ }^{11}$ On the other hand, $1.5-2 \mathrm{~cm}$ of the tumor-free vaginal cuff is routinely resected in clinical practice. ${ }^{8}$

Some factors have been reported to be prognostic in $\mathrm{RH}$, including tumor size, FIGO stage, nodal status, LVSI, depth of stromal invasion, parametrial involvement, histological type, and adjuvant therapy. ${ }^{12-15}$ However, most of the current studies focused on pelvic node metastasis and parametrial invasion; consequently, the knowledge of the VCL to be removed during $\mathrm{RH}$ is still limited. Hence, the rationale and role of VCL resection in the prognosis of cervical cancer are less clear. A too short vaginal resection may lead to local recurrence, while excessive removal of the vagina may cause unnecessary complications and seriously reduce the postoperative quality of life. ${ }^{16}$ So, how much VCL should be resected in order to balance the complications and recurrence? The objective of this study was to reveal the association of VCL with DFS, OS, and local recurrence and distant metastasis rates in patients with cervical cancer following $\mathrm{RH}$.

\section{Methods}

\section{Study design and patients}

This was a retrospective study that compared long-term survival outcomes of patients with cervical cancer (FIGO stage IB-IIA) who underwent RH with VCL $\leq 2.0 \mathrm{~cm}$ vs VCL $>2.0 \mathrm{~cm}$ from January 2007 to February 2017 in Zhongnan Hospital (University Hospital), the Second Clinical College of Wuhan University (Wuhan, People's Republic of China).

The study was carried out according to the Declaration of Helsinki, and informed consent was obtained from all the patients before treatment. Ethical approval was obtained from the Institutional Review Board of the Zhongnan Hospital of Wuhan University.

We hypothesized that VCL during $\mathrm{RH}$ is a prognostic factor for cervical cancer and patients with longer VCL have a better survival outcome and a lower rate of recurrence.
To minimize the bias related to surgeons, only the data of patients operated by five senior consultant surgeons with more experience in gynecological oncology procedures were collected. All the patients were reevaluated by two experienced surgeons 1 day before surgery to confirm the FIGO stage.

Clinical and histological data of all the involved patients who underwent surgical treatment were reviewed from patient medical records by two attending physicians. The information included patient demographics, FIGO stage, surgical procedures, tumor histology and grade, lymph node counts and status, tumor size, VCL, margin status, LVSI, parametrial involvement, depth of stromal invasion, use of adjuvant therapy, and follow-up data.

The eligibility criteria for the analyses were as follows: 1) histologically proven cervical cancer of SCC, AC, or ASC; 2) RH with bilateral pelvic and/or para-aortic lymphadenectomy; and 3) negative margin status of surgical specimens. Patients who underwent chemotherapy or radiotherapy before surgery or who received surgical treatment other than RH were excluded. A total of 1355 patients underwent surgical treatment. Of these, 646 patients received chemotherapy or radiotherapy before surgery, while 16 patients received surgical treatment other than RH; further, histological types other than SCC, AC, or ASC were confirmed in 63 patients, 58 patients showed positive margin status, and 137 patients had incomplete data of VCL. Finally, 435 patients were included in this study (Figure 1).

As this was a retrospective study, perfect matching was not possible. Nevertheless, we still compared the two groups according to age, FIGO stage, tumor size, tumor grade, lymph node status, parametrial involvement, LVSI, histological type, and adjuvant therapy.

\section{Histology}

Histopathological types were categorized according to the WHO classification. The depth of tumor invasion was categorized by the proportion of the cervical wall invasion by the tumor as medial one-half and lateral one-half. LVSI was defined as the presence of neoplastic cells within endothelium-lined spaces. The histopathological diagnosis of the surgical specimen was completed and confirmed by at least two pathologists. Moreover, for the purpose of this study, all the pathological slides were reevaluated blindly by another experienced gynecological pathologist.

\section{Measurement of VCL and tumor size}

After RH, the surgical specimens were handled in a standardized manner immediately. An attending surgeon made a 


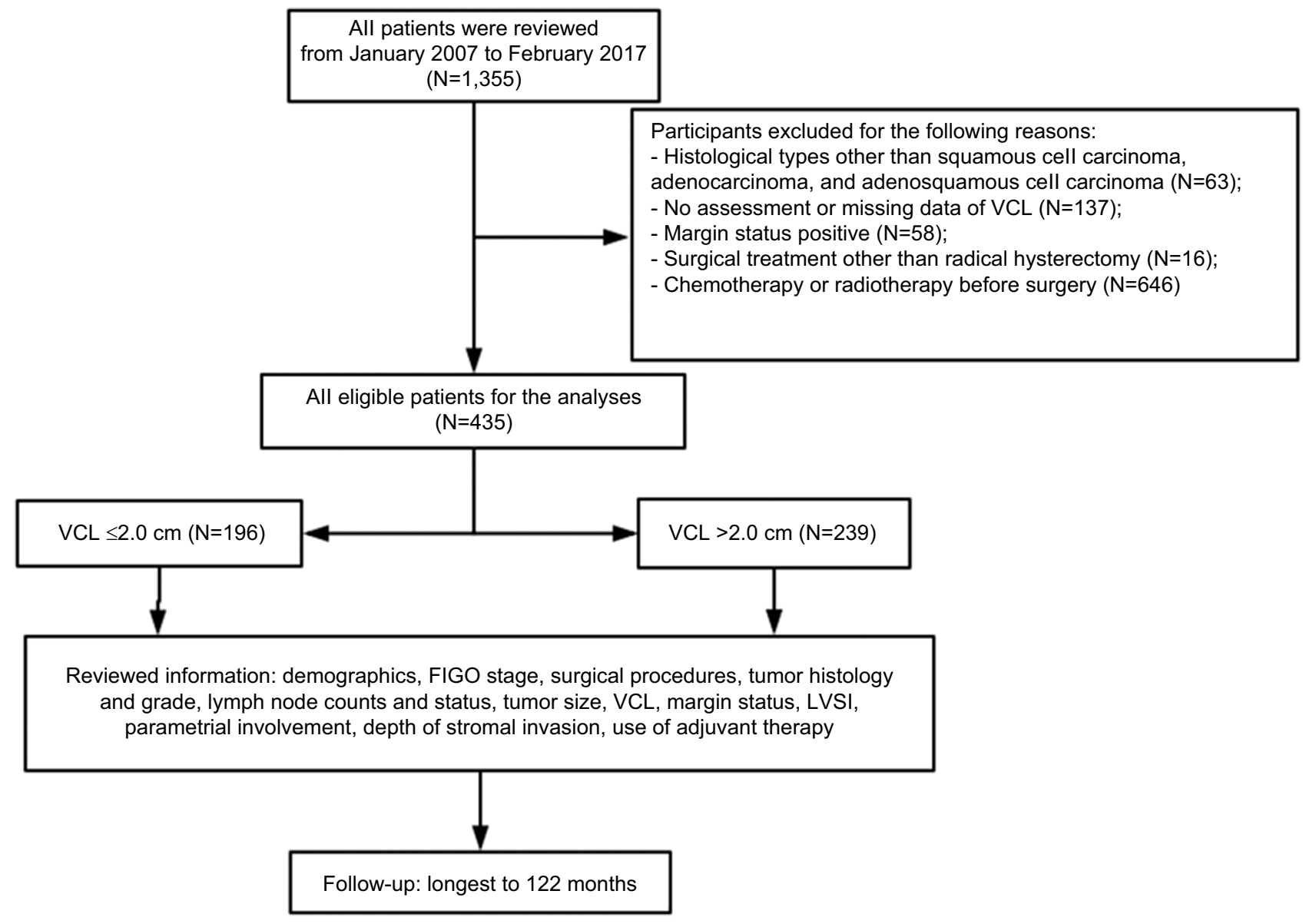

Figure I Study selection process.

Abbreviations: FIGO, International Federation of Gynecology and Obstetrics; LVSI, lymphovascular space invasion; VCL, vaginal cuff length.

ventral Y-shaped incision from the uterine fundus to the vaginal cuff on the specimen, and the VCL and tumor size were then measured. The VCL was measured on a fresh sample without stretching; it is the minimum distance between the edge of the cervix or tumor (the edge of the cervix for stage IB and the edge of tumor for stage IIA) and resection margin.

\section{Patient follow-up}

All patients were followed up every 3 months in the first 2 years and then subsequently every 6 months. Pelvic and cytological examinations were performed at every visit. CT or MRI was performed in every 12 months or when clinically indicated. The end point was the time from the start of surgery to the earliest diagnosis of recurrence or death.

\section{Study outcomes}

The primary end points of this study were local recurrence, distant metastasis, DFS, and OS. The time to local recurrence was calculated from the date of surgery to the date of first documented primary recurrence. The time to distant metastasis was calculated from the date of surgery to the date of first documented distant metastasis. DFS was defined as the time from the date of surgery to the date of death or recurrence, either local recurrence or distant metastasis. OS was computed from the date of surgery to the date of death or last follow-up.

\section{Recurrence assessment}

Recurrence case was diagnosed during the regular followup visit and mainly confirmed by $\mathrm{CT}$ and/or MRI. Whenever possible, histological or cytological confirmation was obtained. Sites of recurrence were classified as local and distant. Local recurrence included central (ie, around the vaginal stump or paracolpium) or pelvic sidewall. Distant metastasis was defined as tumor recurrence at a site beyond the pelvic radiation field. Para-aortic lymph 
node or other distant node metastases were defined as distant metastases.

\section{Statistical analysis}

Participants and tumor characteristics were grouped according to VCL. Chi-squared tests or Fisher's exact tests were used to compare the categorical variables between the VCL groups. Student's $t$-tests were used to compare continuous variables such as age and number of lymph nodes. The survival outcomes (local recurrence, distant metastasis, DFS, and OS) were estimated according to VCL and compared using the Kaplan-Meier method and log-rank statistic, respectively. Cox proportional hazards regression models were used to estimate the relative likelihood of DFS and OS with various factors in both univariate and multivariate analyses. Variables with a probability value $<0.10$ were included in a Cox multivariate proportional hazards regression analysis. Several additional variables were selected for the multivariate analysis according to literature (ie, LVSI and histological type). The results are presented as HRs with $95 \%$ CIs. A $P$-value of $<0.05$ was considered to be statistically significant. Statistical analysis was performed using the SPSS 16.0 statistical package (version 16.0; SPSS Inc., Chicago, IL, USA).

\section{Results}

\section{Clinical and pathological characteristics}

Table 1 presents the clinical and pathological characteristics of the included patients. The median age at diagnosis was 44 years (range 21-68 years), and the median follow-up time was 60 months (range 12-122 months). Of the included patients, $269(61.8 \%)$ were diagnosed as having FIGO stage IB cervical cancer, and 367 (84.4\%) had squamous cell carcinoma. The median number of lymph nodes removed was 24 (range 12-45), and lymph node positive rate was $18.6 \%$. Forty-six patients $(10.6 \%)$ had parametrial involvement, and LVSI was present in 164 patients $(37.7 \%)$. In $>50 \%$ of the patients, the depth of stromal invasion was $\leq 1 / 2$. Adjuvant therapy was administered in $47.4 \%$ of the patients, and $45-50$ Gy of external beam pelvic radiation therapy was administered to the patients who underwent chemoradiotherapy or radiotherapy alone followed by surgery.

The patients with a longer VCL were more likely to be in an advanced FIGO stage $(P=0.031)$. Moreover, patients with VCL $>2.0 \mathrm{~cm}$ showed a significant higher depth of stromal invasion than patients with VCL $\leq 2.0 \mathrm{~cm}(P=0.001)$. In addition, in the group of longer VCL, parametrial involvement was also more commonly observed $(P=0.035)$. Around $50 \%$ $(120 / 239)$ of patients with VCL $>2.0 \mathrm{~cm}$ received adjuvant therapy as compared to $44 \%(86 / 196)$ of those with VCL $\leq 2.0$ $\mathrm{cm}$. The distribution of other clinicopathological characteristics (tumor grade, maximal tumor size, and histological type) was not statistically associated with VCL.

\section{Survival analysis based on VCL}

There were $60(30.6 \%)$ vs $28(11.7 \%)$ recurrences $(P<0.001)$ and $53(27.0 \%)$ vs $22(9.2 \%)$ deaths $(P<0.001)$ in the VCL $\leq 2.0 \mathrm{~cm}$ and $\mathrm{VCL}>2.0 \mathrm{~cm}$ groups, respectively. We analyzed the survival data of these patients by the Kaplan-Meier analysis to understand the clinical significance of VCL in RH. For all the 435 patients with cervical cancer, the overall 5-year DFS and OS were 78.7\% (95\% CI: 74.4\%-83.0\%) and $81.1 \%$ (95\% CI: $77.0 \%-85.2 \%)$, respectively.

In the VCL $>2.0 \mathrm{~cm}$ group, the 5-year DFS was $87.5 \%$, which was significantly higher than that of the VCL $\leq 2.0 \mathrm{~cm}$ group (68.1\%; $P<0.001$; Figure 2A). Similarly, the 5-year OS was $89.2 \%$ and $71.4 \%$ for the VCL $>2.0 \mathrm{~cm}$ and VCL $\leq 2.0$ cm groups, respectively ( $P<0.001$; Figure $2 \mathrm{~B})$. We also used the Kaplan-Meier analysis to assess the impact of VCL on postoperative local and distant recurrence rate. The 5-year local recurrence rate was $10.9 \%$ and $26.9 \%$ for the VCL $>2.0 \mathrm{~cm}$ and VCL $\leq 2.0 \mathrm{~cm}$ groups, respectively $(P<0.001$; Figure 2C), while the 5-year distant metastasis rate was $5.0 \%$ and $6.9 \%$ for the VCL $>2.0 \mathrm{~cm}$ and $\mathrm{VCL} \leq 2.0 \mathrm{~cm}$ groups, respectively ( $P=0.212$; Figure $2 \mathrm{D})$.

\section{Univariate and multivariate Cox regression analysis}

Univariate analysis suggested that VCL, FIGO stage, maximal tumor size, depth of stromal invasion, positive lymph node status, parametrial involvement, and adjuvant therapy were significantly associated with both DFS and OS (Table 2).

For the multivariate Cox proportional hazards models, the variates were selected according to the univariate analyses. Consequently, as shown in Table 3 , independent prognostic factors of cervical cancer for DFS were VCL $\leq 2.0 \mathrm{~cm}$ (HR 4.344 [95\% CI: 2.695-7.000]), FIGO stage (HR 4.942 [95\% CI: $2.467-9.898]$ ), and positive lymph node status (HR 3.711 [95\% CI: 2.383-5.778]). Further, the independent prognostic factors in the multivariate analysis for OS were VCL $\leq 2.0 \mathrm{~cm}$ (HR 4.830 [95\% CI: 2.849-8.190]), FIGO stage IIA2 (HR 5.228[95\% CI: 2.487 - 10.989]), and positive lymph node status (HR 4.021 [95\% CI: 2.449-6.472]).

\section{Discussion}

In our study, we found that VCL was strongly associated with 5-year DFS and OS in patients with cervical cancer (FIGO 
Table I Summary of clinical and pathological characteristics $(n=435)$

\begin{tabular}{|c|c|c|c|c|}
\hline \multirow[t]{2}{*}{ Characteristics } & \multicolumn{2}{|l|}{$\operatorname{VCL}(\mathrm{cm})$} & \multirow{2}{*}{$\begin{array}{l}\text { Mean or } \\
\text { total }\end{array}$} & \multirow[t]{2}{*}{$P$-value } \\
\hline & $\leq 2.0 \mathrm{~cm}(\mathrm{~N}=196)$ & $>2.0 \mathrm{~cm}(\mathrm{~N}=239)$ & & \\
\hline \multicolumn{5}{|l|}{ Age (years) } \\
\hline Mean (range) & $45.5(2 I-67)$ & $44.2(2 I-68)$ & $44.80(21-68)$ & 0.290 \\
\hline \multicolumn{5}{|l|}{ Number of lymph node } \\
\hline Mean (range) & $25.3(12-45)$ & $24.6(12-45)$ & $24.9(12-45)$ & 0.313 \\
\hline \multicolumn{5}{|l|}{ FIGO stage, n (\%) } \\
\hline Stage IBI & $64(32.7)$ & $54(22.6)$ & II 8 (27.I) & 0.031 \\
\hline Stage IB2 & $69(35.2)$ & $82(34.3)$ & I5I (34.7) & \\
\hline Stage IIAI & $45(23.0)$ & $64(26.8)$ & $109(25.1)$ & \\
\hline Stage IIA2 & $18(9.2)$ & $39(16.3)$ & $57(13.1)$ & \\
\hline \multicolumn{5}{|l|}{ Tumor grade, n (\%) } \\
\hline GI & $51(26.0)$ & $56(23.4)$ & $107(24.6)$ & 0.822 \\
\hline G2 & $90(45.9)$ & $114(47.7)$ & $204(46.9)$ & \\
\hline G3 & $55(28.1)$ & $69(28.9)$ & $124(28.5)$ & \\
\hline \multicolumn{5}{|l|}{ Maximal tumor size, n (\%) } \\
\hline$<2 \mathrm{~cm}$ & $4 \mathrm{I}(20.9)$ & $58(24.3)$ & $99(22.8)$ & 0.055 \\
\hline $2-4 \mathrm{~cm}$ & $70(35.7)$ & $60(25.1)$ & $130(29.9)$ & \\
\hline$>4 \mathrm{~cm}$ & $85(43.4)$ & $|2|(50.6)$ & $206(47.4)$ & \\
\hline \multicolumn{5}{|c|}{ Depth of stromal invasion, $\mathrm{n}(\%)$} \\
\hline$\leq 1 / 2$ & II (59.7) & $104(43.5)$ & $221(50.8)$ & 0.001 \\
\hline$>1 / 2$ & $79(40.3)$ & $135(56.5)$ & $214(49.2)$ & \\
\hline \multicolumn{5}{|l|}{ Lymph node status, n (\%) } \\
\hline Negative & $165(84.2)$ & $189(79.1)$ & $354(8 I .4)$ & 0.174 \\
\hline Positive & $3 I(I 5.8)$ & $50(20.9)$ & $81(18.6)$ & \\
\hline \multicolumn{5}{|l|}{ Parametrial involvement, n (\%) } \\
\hline No & $182(92.9)$ & $207(86.6)$ & 389 (89.4) & 0.035 \\
\hline Yes & $14(7.1)$ & $32(13.4)$ & $46(10.6)$ & \\
\hline \multicolumn{5}{|l|}{ LVSI, n (\%) } \\
\hline No & $13 \mid(66.8)$ & $140(58.6)$ & $27 \mid(62.3)$ & 0.077 \\
\hline Yes & $65(33.2)$ & $99(4 \mid .4)$ & $164(37.7)$ & \\
\hline \multicolumn{5}{|l|}{ Histology, n (\%) } \\
\hline SCC & $168(85.7)$ & $199(83.3)$ & $367(84.4)$ & 0.484 \\
\hline AC or ASC & $28(14.3)$ & $40(16.7)$ & $68(15.6)$ & \\
\hline \multicolumn{5}{|l|}{ Adjuvant therapy, n (\%) } \\
\hline No & $110(56.1)$ & $119(49.8)$ & $229(52.6)$ & 0.188 \\
\hline Yes & $86(43.9)$ & $120(50.2)$ & $206(47.4)$ & \\
\hline \multicolumn{5}{|l|}{ Recurrence disease, $\mathrm{n}(\%)$} \\
\hline Locoregional only & $46(76.7)$ & $16(57.1)$ & $62(70.5)$ & 0.008 \\
\hline Distant only & II (18.3) & $4(14.3)$ & $15(17.0)$ & \\
\hline Locoregional plus distant & $3(5.0)$ & $8(28.6)$ & II (12.5) & \\
\hline
\end{tabular}

Abbreviations: AC, adenocarcinoma; ASC, adenosquamous carcinoma; FIGO, International Federation of Gynecology and Obstetrics; LVSI, lymphovascular space invasion; SCC, squamous cell carcinoma; VCL, vaginal cuff length.

stage IB-IIA). The 5-year DFS and OS were significantly higher in women with $\mathrm{VCL}>2 \mathrm{~cm}(P<0.001)$. Moreover, the 5 -year local recurrence rate was also significantly lower in women with $\mathrm{VCL}>2 \mathrm{~cm}(P<0.001)$. However, there was no significant association between the 5-year distant metastasis rate and VCL $(P=0.212)$. Our findings are consistent with prior qualitative studies which reported that the closer margin status was associated with higher rate of recurrence or local recurrence as shown by Viswanathan et al; their survey also showed that the relapse-free survival rate was increased with each millimeter increase in distance from the tumor. ${ }^{17}$ Similarly, another study reported that surgical margins of $<5$ $\mathrm{mm}$ are often associated with recurrence in the presence of other high- or intermediate-risk factors. ${ }^{18}$ Though the primary focus of the above studies was on surgical margin rather than on VCL, their conclusions may provide an explanation for our results.

On the other hand, in contrast to our findings, Kim et al reported in 2011 that VCL was not associated with vaginal and pelvic recurrence rates in patients with cervical cancer 

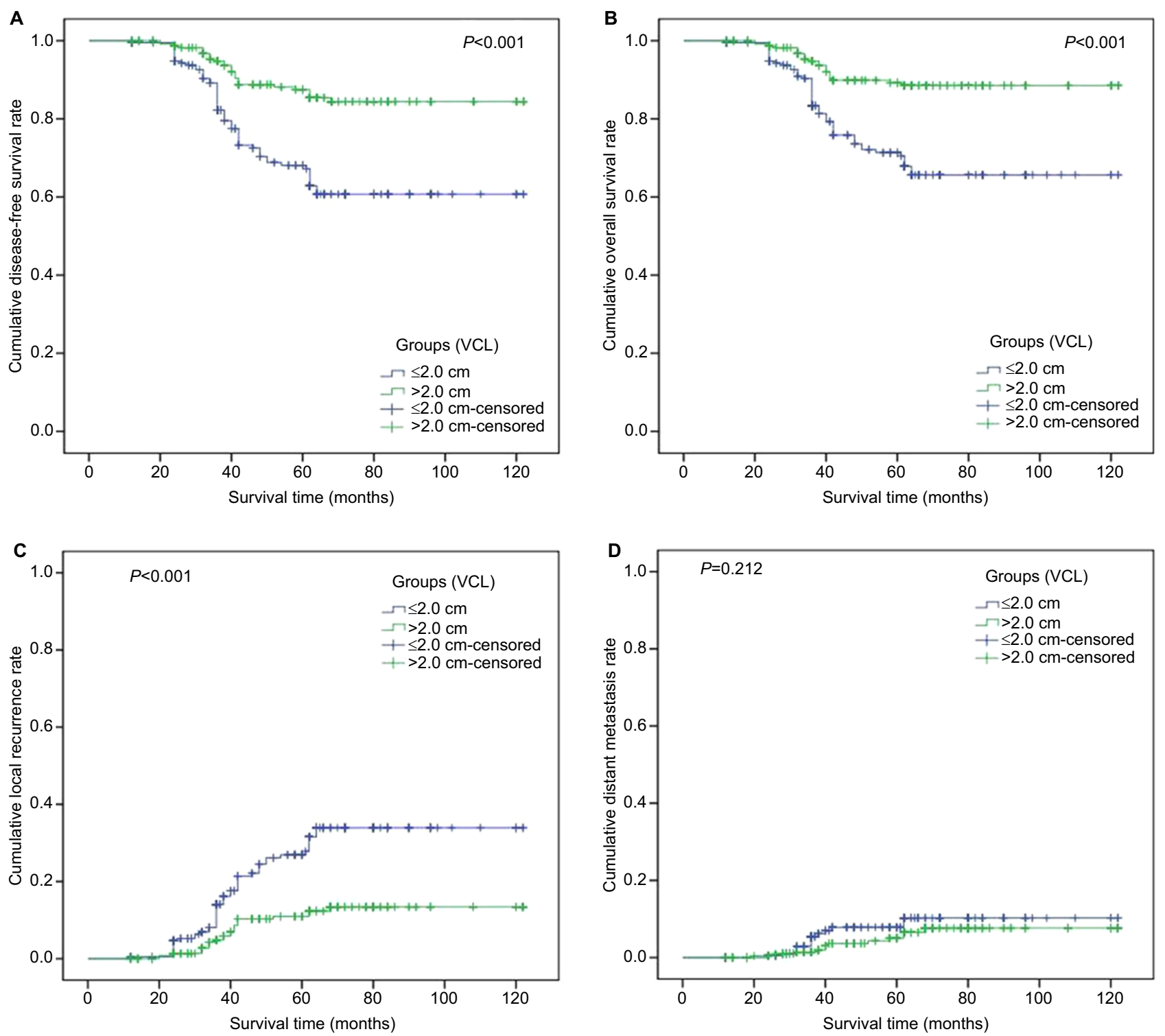

Figure 2 Kaplan-Meier survival curves showing the correlation of VCL with long-term survival in patients with cervical cancer (FIGO stage IB-IIA). Notes: (A) Disease-free survival; (B) overall survival; (C) local recurrence rate; (D) distant metastasis rate. Abbreviations: FIGO, International Federation of Gynecology and Obstetrics; VCL, vaginal cuff length.

following RH. ${ }^{10}$ They assessed the 3-year vaginal and pelvic recurrence rate in 280 Korean women with a majority of them having stage IB1 (73.2\%) cervical cancer and $68 \%$ of the participants had received adjuvant therapy. A more recent study conducted by Edwards et al in 2015 also reported that VCL was not associated with vaginal and pelvic recurrence among patients with cervical cancer. They analyzed the data of 169 patients with stage IB1 cervical cancer with a median follow-up time of 12 years. ${ }^{19}$ Both these studies primarily evaluated patients with stage IB1 disease, and their end result was vaginal and pelvic recurrence. Results of the present study may have been affected by a greater proportion of patients with more advanced disease (stage IB2-IIA2, 73\%), a lower percent of patients received adjuvant therapy, and a relatively longer follow-up period (up to 10 years). These might be the potential reasons that explain the association between VCL and local recurrence, DFS, and OS.

Currently, there is no uniform standard for the length of vaginal resection during RH. Different surgical classification methods of RH are also controversial about the recommended length of vaginal resection. The classic Piver et al classification, published in 1974, described five classes of extended hysterectomies for women with cervical cancer, in which the vaginal resection was excessive (one-third to three-quarters of the vagina). ${ }^{8}$ In addition, the current updated Querleu-Morrow classification of RH is based only on the lateral extent of para- 
Table 2 Univariate Cox regression analysis of prognostic factors of cervical cancer

\begin{tabular}{|c|c|c|c|c|c|c|}
\hline \multirow[t]{2}{*}{ Variables } & \multicolumn{3}{|l|}{ DFS } & \multicolumn{3}{|l|}{ OS } \\
\hline & HR & $95 \% \mathrm{Cl}$ & $P$-value & HR & $95 \% \mathrm{Cl}$ & $P$-value \\
\hline Age, I-year increment & 0.989 & $0.972-1.005$ & 0.169 & 0.989 & $0.972-1.007$ & 0.245 \\
\hline $\mathrm{VCL}>2.0 \mathrm{~cm}$ & Ref. & & & Ref. & & \\
\hline $\mathrm{VCL} \leq 2.0 \mathrm{~cm}$ & 2.940 & $1.876-4.608$ & $<0.001$ & 3.207 & $1.949-5.275$ & $<0.001$ \\
\hline Stage IBI & Ref. & & & Ref. & & \\
\hline Stage IB2 & 2.288 & I.209-4.328 & 0.011 & 2.225 & I. $120-4.210$ & 0.022 \\
\hline Stage IIAI & 2.195 & I.I27-4.273 & 0.021 & 1.882 & $0.905-3.912$ & 0.090 \\
\hline Stage IIA2 & 4.193 & $2.129-8.257$ & $<0.001$ & 4.313 & $2.090-8.899$ & $<0.001$ \\
\hline Grade GI & Ref. & & & & & \\
\hline Grade G2 & 1.304 & $0.760-2.239$ & 0.335 & 1.495 & $0.808-2.765$ & 0.200 \\
\hline Grade G3 & 1.182 & $0.654-2.137$ & 0.579 & 1.505 & $0.778-2.910$ & 0.224 \\
\hline Size $<2 \mathrm{~cm}$ & Ref. & & & Ref. & & \\
\hline Size $2-4 \mathrm{~cm}$ & 2.822 & $1.340-5.946$ & 0.006 & 2.949 & $1.270-6.845$ & 0.012 \\
\hline Size $>4 \mathrm{~cm}$ & 3.443 & $1.689-7.018$ & 0.001 & 3.979 & I.790-8.846 & 0.001 \\
\hline Depth of stromal invasion & 1.667 & $1.092-2.547$ & 0.018 & 2.005 & $1.256-3.203$ & 0.004 \\
\hline Lymph node status & 3.322 & $2.166-5.095$ & $<0.001$ & 3.514 & $2.218-5.569$ & $<0.001$ \\
\hline Parametrial involvement & 2.383 & $1.403-4.047$ & 0.001 & 2.505 & $1.422-4.414$ & 0.001 \\
\hline LVSI & 1.114 & $0.727-1.708$ & 0.620 & 1.175 & $0.742-1.860$ & 0.493 \\
\hline Histology & 1.387 & $0.826-2.328$ & 0.216 & 1.342 & $0.762-2.363$ & 0.308 \\
\hline Adjuvant therapy & 1.619 & $1.056-2.482$ & 0.027 & 1.719 & $1.080-2.737$ & 0.022 \\
\hline
\end{tabular}

Abbreviations: DFS, disease-free survival; LVSI, lymphovascular space invasion; OS, overall survival; VCL, vaginal cuff length.

Table 3 Multivariate analysis of prognostic factors of cervical cancer

\begin{tabular}{|c|c|c|c|c|c|c|}
\hline \multirow[t]{2}{*}{ Variables } & \multicolumn{3}{|l|}{ DFS } & \multicolumn{3}{|l|}{ OS } \\
\hline & HR & $95 \% \mathrm{Cl}$ & $P$-value & HR & $95 \% \mathrm{Cl}$ & $P$-value \\
\hline $\mathrm{VCL}>2.0 \mathrm{~cm}$ & Ref. & & & Ref. & & \\
\hline$V C L \leq 2.0 \mathrm{~cm}$ & 4.344 & $2.695-7.000$ & $<0.001$ & 4.830 & $2.849-8.190$ & $<0.001$ \\
\hline Stage IBI & Ref. & & & Ref. & & \\
\hline Stage IB2 & 1.981 & $1.043-3.763$ & 0.037 & 1.918 & $0.96 \mathrm{I}-3.829$ & 0.065 \\
\hline Stage IIAI & 2.149 & I.092-4.228 & 0.027 & 1.800 & $0.857-3.780$ & 0.121 \\
\hline Stage IIA2 & 4.942 & $2.467-9.898$ & $<0.001$ & 5.228 & $2.487-10.989$ & $<0.001$ \\
\hline Lymph node status & 3.711 & $2.383-5.778$ & $<0.001$ & 4.021 & $2.499-6.472$ & $<0.001$ \\
\hline
\end{tabular}

Abbreviations: DFS, disease-free survival; OS, overall survival; VCL, vaginal cuff length.

metrium resection, which proposed four types (A-D) of $\mathrm{RH}$ with detailed description on the lateral resection part, but not a clear recommendation for VCL. ${ }^{8,11}$ In Type $\mathrm{C}$ that is indicated for FIGO stage IB-IIA, they have proposed "the length of the vaginal cuff is adjusted to the vaginal extent of the tumor". ${ }^{11}$

There should also be an optimal range for the length of the vaginal resection during RH. A study on TMMR, in which the embryologically defined uterovaginal compartment was removed in patients with cervical cancer (stage IB-IIA and selected IIB), found that TMMR is pivotal in pelvic control. ${ }^{4}$ Though not conclusive, as a part of the TMMR, the recommended safe margin for vaginal cuff resection was 1-1.5 $\mathrm{cm}$. However, Zullo et al reported that significantly longer length of vaginal resection in RH was associated with detrusor dysfunction, thereby causing urine storage and voiding problem. ${ }^{16}$ In our study, we consider that the most appropriate VCL could be $>2 \mathrm{~cm}$ for cervical cancer (FIGO stage IBIIA). However, our study was unable to determine an optimal ceiling for VCL, which is a limitation of this study.

Apart from VCL, we also identified FIGO stage and lymph node involvement as significant prognostic factors for cervical cancer. It is known that the locally advanced stage is an unfavorable prognostic factor of cervical cancer. ${ }^{20}$ Moreover, recent studies have reported that the FIGO stage was an independent prognostic factor for the risk of both recurrence and death. ${ }^{21,22}$ In our study, patients with stage II cervical cancer showed significantly unfavorable OS (HR 2.320 [95\% CI: 1.438-3.745]) compared to patients in stage I (data not shown).

This is a single institution-based retrospective study; and hence, some limitations are present. There might be other 
confounding variables apart from VCL that could also have played a role in prognosis and recurrence. Apart from possible clinicopathological factors, other confounding factors include age, height, weight, and menopause. These factors may influence the measurement of vaginal length. ${ }^{23} \mathrm{~A}$ large number of patients were excluded from the analysis because of having incomplete data. In addition, as histological biopsies are invasive procedures and require informed consent from patients, only a small number of patients agreed to receive histological examination. Despite these limitations, this is a large comprehensive study with encouraging result to evaluate the 5-year DFS and OS for patients with cervical cancer (stage IB-IIA) based on VCL following RH.

\section{Conclusion}

VCL is an important prognostic factor, and VCL $>2 \mathrm{~cm}$ is a favorable factor for DFS and OS in patients with cervical cancer (FIGO stage IB-IIA). In addition, VCL $<2 \mathrm{~cm}$ also significantly increases the risk of local recurrence of cervical cancer. A well-designed multicenter study with an adequate sample size is recommended to validate VCL as a prognostic factor and to define the VCL cutoff value in RH for cervical cancer (FIGO stage IB-IIA).

\section{Abbreviations}

$\mathrm{AC}$, adenocarcinoma

ASC, adenosquamous carcinoma

CT, computed tomography

DFS, disease-free survival

FIGO, International Federation of Gynecology and Obstetrics

LSVI, lymphovascular space invasion

MRI, magnetic resonance imaging

OS, overall survival

$\mathrm{RH}$, radical hysterectomy

SCC, squamous cell carcinoma

TMMR, total mesometrial resection

VCL, vaginal cuff length

\section{Acknowledgments}

The authors acknowledge all the participants of this study. The authors also acknowledge Zhongnan Hospital of Wuhan University and the staff from the Department of Gynecological Oncology for assistance during data collection. The authors are grateful to the National Natural Science Foundation of China for providing logistic support for this study. Moreover, the authors also appreciate Professor Marcia A Petrini (Chiang Mai University, Faculty of Nursing) for her contribution to language editing of the paper. This study was funded by the National Natural Science Foundation of China (grant no. 81272866).

\section{Data sharing statement}

The data sets used and analyzed during the current study are available from the corresponding author on reasonable request.

\section{Disclosure}

The authors report no conflicts of interest in this work.

\section{References}

1. Torre LA, Bray F, Siegel RL, Ferlay J, Lortet-Tieulent J, Jemal A. Global cancer statistics, 2012. CA Cancer J Clin. 2015;65(2):87-108.

2. Lee JH, Lee SW, Kim JR, et al. Tumour size, volume, and marker expression during radiation therapy can predict survival of cervical cancer patients: a multi-institutional retrospective analysis of KROG 16-01. Gynecol Oncol. 2017;147(3):577-584.

3. Chen W, Zheng R, Baade PD. Cancer statistics in China, 2015. CA Cancer J Clin. 2016;66(2):115-132.

4. Höckel M, Horn LC, Manthey N, et al. Resection of the embryologically defined uterovaginal (Müllerian) compartment and pelvic control in patients with cervical cancer: a prospective analysis. Lancet Oncol. 2009;10(7):683-692.

5. Quinn MA, Benedet JL, Odicino F, et al. Carcinoma of the cervix uteri. FIGO 26th Annual Report on the Results of Treatment in Gynecological Cancer. Int J Gynaecol Obstet. 2006;95(Suppl 1):S43-S103.

6. Saatli B, Olgan S, Gorken IB, et al. Tumor-free distance from outermost layer of cervix is of prognostic value in surgically treated cervical cancer patients: a multicenter study. Arch Gynecol Obstet. 2014;289(6):1331-1335.

7. Swailes AL, Gockley A, Phaeton R, Kesterson JP. The Wertheim hysterectomy: Development, modifications, and impact in the present day. Gynecol Oncol. 2017;145:3-8.

8. Querleu D, Morrow CP. Classification of radical hysterectomy. Lancet Oncol. 2008;9(3):297-303.

9. Piver MS, Rutledge F, Smith JP. Five classes of extended hysterectomy for women with cervical cancer. Obstet Gynecol. 1974;44(2):265-272.

10. Kim K, Cho SY, Park SI, et al. Vaginal and pelvic recurrence rates based on vaginal cuff length in patients with cervical cancer who underwent radical hysterectomies. Eur J Surg Oncol. 2011;37(9):824-827.

11. Querleu D, Cibula D, Abu-Rustum NR. 2017 Update on the QuerleuMorrow Classification of Radical Hysterectomy. Ann Surg Oncol. 2017;24(11):3406-3412.

12. Zreik TG, Chambers JT, Chambers SK. Parametrial involvement, regardless of nodal status: a poor prognostic factor for cervical cancer. Obstet Gynecol. 1996;87(5 Pt 1):741-746.

13. Herr D, König J, Heilmann V, Koretz K, Kreienberg R, Kurzeder C. Prognostic impact of satellite-lymphovascular space involvement in early-stage cervical cancer. Ann Surg Oncol. 2009;16(1):128-132.

14. Delgado G, Bundy B, Zaino R, Sevin BU, Creasman WT, Major F. Prospective surgical-pathological study of disease-free interval in patients with stage IB squamous cell carcinoma of the cervix: a Gynecologic Oncology Group study. Gynecol Oncol. 1990;38(3):352-357.

15. Xia X, Xu H, Wang Z, Liu R, Hu T, Li S. Analysis of Prognostic Factors Affecting the Outcome of Stage IB-IIB Cervical Cancer Treated by Radical Hysterectomy and Pelvic Lymphadenectomy. Am J Clin Oncol. 2016;39(6):604-608.

16. Zullo MA, Manci N, Angioli R, Muzii L, Panici PB. Vesical dysfunctions after radical hysterectomy for cervical cancer: a critical review. Crit Rev Oncol Hematol. 2003;48(3):287-293.

17. Viswanathan AN, Lee H, Hanson E, Berkowitz RS, Crum CP. Influence of margin status and radiation on recurrence after radical hysterectomy in Stage IB cervical cancer. Int J Radiat Oncol Biol Phys. 2006;65(5):1501-1507. 
18. McCann GA, Taege SK, Boutsicaris CE, et al. The impact of close surgical margins after radical hysterectomy for early-stage cervical cancer. Gynecol Oncol. 2013;128(1):44-48.

19. Edwards AM, Edwards J, Baldwin LA, Desimone CP. Does vaginal buff length after radical hysterectomy predict a localized recurrence of cervical cancer? [236]. Obstet Gynecol. 2015;125(Suppl. 1):S76. Available from: https://journals.lww.com/greenjournal/Abstract/2015/05001/Does_Vaginal_Cuff_Length_After_Radical.258.aspx. Accessed October 25, 2018.

20. Hong JH, Tsai CS, Chang JT, et al. The prognostic significance of preand posttreatment SCC levels in patients with squamous cell carcinoma of the cervix treated by radiotherapy. Int J Radiat Oncol Biol Phys. 1998;41(4):823-830.
21. Kimyon Comert G, Turkmen O, Karalok A, Basaran D, Bulbul D, Turan T. Therapy Modalities, Prognostic Factors, and Outcome of the Primary Cervical Carcinosarcoma: Meta-analysis of Extremely Rare Tumor of Cervix. Int J Gynecol Cancer. 2017;27(9):1957-1969.

22. Yang SS, Gao Y, Wang DY, et al. Overexpression of eukaryotic initiation factor 5A2 (EIF5A2) is associated with cancer progression and poor prognosis in patients with early-stage cervical cancer. Histopathology. 2016;69(2):276-287.

23. Tan JS, Lukacz ES, Menefee SA, Luber KM, Albo ME, Nager CW. Determinants of vaginal length. Am J Obstet Gynecol. 2006;195(6) $1846-1850$
Cancer Management and Research

\section{Publish your work in this journal}

Cancer Management and Research is an international, peer-reviewed open access journal focusing on cancer research and the optimal use of preventative and integrated treatment interventions to achieve improved outcomes, enhanced survival and quality of life for the cancer patient The manuscript management system is completely online and includes

\section{Dovepress}

a very quick and fair peer-review system, which is all easy to use. Visit http://www.dovepress.com/testimonials.php to read real quotes from published authors. 